\title{
Conclusion : alliances, résonances, trajectoires
}

Lénigme est au centre de l'œuvre d'Esther Tellermann. Or, ses poèmes ne sont tendus vers l'épiphanie d'aucun Être poétique, ${ }^{1}$ mais plutôt vers la langue dans sa matérialité et sa sensualité. Imaginons quelqu'un méditant ses textes, qui en vient à se demander : Qui suis-je, moi, m'immergeant dans cet univers? Qui sommes-nous, ses lecteurs et lectrices, explorant ensemble cet inconscient ? S'y baigner, est-ce une caresse ou un crime $(C E)$, la sensualité d'une découverte d'ordre symbolique ou le danger parfois délicieux d'un pas vers des zones d'ombre? Dans quel but poursuit-on ce dialogue inlassable avec l'Autre ? Pourquoi une telle intensité dans l'évocation du renouveau des rites et des symboles partagés, d'une quête identitaire communautaire et universelle, des alliances avec la langue et autrui qui font éclore l'à-venir?

L'ouvre tellermannienne nous encourage à nous poser de telles questions. Mais, mieux encore, elle les rend immédiates, à tout moment. Comme le dit l'auteur, l'écriture poétique est «un acte de pur présent», « geste » qui peut « imprimer dans le vers le plus ténu l'amplitude de l'univers $»^{2}$ C'est un savoir qui surgit avant que la raison n'intervienne, énigmatique «autant dans son émergence que sa jouissance ${ }^{3}$ Tellermann fait partie intégrante de la poésie de l'après-guerre et de l'extrême contemporain parce qu'elle fait sentir cette émergence et cette jouissance tout en puisant dans l'Histoire et en dialoguant avec ses semblables. On peut remarquer ce qu'André du Bouchet appelle la «fraîcheur» qui émane lorsque le langage «ne se referme pas sur soi » ${ }^{4}$ et, tout à la fois, une ouverture intersubjective singulière, des gestes sacrés, qu'il s'agisse d'appels, de mains tendues, de «bouches à ronces » qui chantent la « déchirure » $(S N 84,78-79)$ ou de gestes encore plus charnels. Là où un Jacques Réda se permettrait de danser avec l'« énergie du monde » dans une «poésie

1 Cf. les vers « toute une famille de regards / se serre dans mes yeux » (Henri Meschonnic, Infiniment à venir, suivi de Pour le poème et par le poème, Orbey: Arfuyen, 2017, p. 33). Nous empruntons la phrase « [ses poèmes] ne sont tendus vers l'épiphanie d'aucun Être poétique » à Gérard Dessons, dans la notice « Henri Meschonnic » du Dictionnaire de poésie de Baudelaire à nos jours, op. cit., p. 489.

2 Anne Malaprade, « Entretien avec Esther Tellermann », op. cit., p. 164-65.

3 Esther Tellermann, « Esther Tellermann : le travail du poète », op. cit., p. 4.

4 André du Bouchet, Entretiens d'André du Bouchet avec Alain Veinstein (1979-200o), op. cit., p. 116. Voir aussi Carnet 2, op. cit., p. 76 : « la poésie n'avance qu'en repoussant l'objet qu'elle entreprend de s'attribuer ».

(C) AARON PREVOTS, 2022 | DOI:10.1163/9789004499683_011 
incarnée entre [ses] bras », ${ }^{5}$ Tellermann offre un regard féminin et tendrement distancié sur la fulgurance du surgissement des signes, les yeux tournés vers des êtres et des choses difficilement situables, vers l'invention d'« une dialectique entre la représentation et la présentation de l'objet aimé et perdu ». ${ }^{6}$

Tout poète se réfère aux auteurs qui l'ont précédé. Dans le cas de Tellermann, la poésie s'incarne en une muse protéiforme. L'inspiration vient à la fois du langage comme fond inépuisable, de l'inconscient dont les plis ne cessent de nous captiver et de la présence de pairs tels que Paul Celan, Saint-John Perse, André du Bouchet, Pierre Reverdy et d'autres encore, à commencer par ceux qui figurent dans Nous ne sommes jamais assez poète. Cette spécificité peut surprendre, mais c'est un aspect clé de l'ensemble des textes. En effet, en ouvrant les mots et les morts, Tellermann ouvre aussi le champ de la poésie française contemporaine. Se ressouvenir, c'est veiller au devenir du langage et au bien-être collectif. S'allier avec les disparus comme le fait Tellermann, par exemple avec Celan et Du Bouchet, c'est garder en mémoire non seulement une vision langagière qui va à l'encontre de « l'universel reportage $»,{ }^{7}$ mais aussi des êtres qui nous sont chers. Est-ce une faiblesse si le cri ne revient pas plus souvent chez Tellermann, une faute malgré soi envers l'histoire juive que tout un chacun peut faire lorsque seule la discrétion semble de mise ${ }^{8}$ ? Cette étude montre de quel respect néanmoins fait preuve l'auteur envers cette histoire et ces traditions en s'adonnant aux sons, aux lettres, aux mots, à la nomination, au déploiement de page en page d'un présent implicitement imprégné de divin et dont il incombe au lecteur de réunir les fragments, voire les étincelles. ${ }^{9}$ Nous avons vu, d'ailleurs, comment Tellermann retrouve implicitement à travers Celan des pratiques talmudiques et kabbalistes. Toute une gamme de

5 Jacques Réda, Celle qui vient à pas légers, Saint Clément : Fata Morgana, 1999 [1985], p. 20, p. 65 .

6 Esther Tellermann, « Esther Tellermann : le travail du poète », op. cit., p. 7 .

7 Ibid. Tellermann cite Stéphane Mallarmé. Cf. «Crise de vers», [Divagations], CEuvres complètes, op. cit., p. 36o-68 (368) : «l'universel reportage dont, la littérature exceptée, participe tout entre les genres d'écrits contemporains » (l'auteur souligne).

8 À propos de cette voix discrète et du phrasé par lequel communique l'auteur, cf. Patrice Beray, «Nous ne sommes jamais assez poète (Esther Tellermann) », Mediapart, 25 janv. 2015, $<$ blogs.mediapart.fr>.

9 Cf. Shira Wolosky, « Mystical Language and Mystical Silence in Paul Celan's "Dein Hinübersein” », Argumentum e Silentio :International Paul Celan Symposium, ed. Amy D. Colin, Berlin/ New York : Walter de Gruyter, 1987, p. 365-74. Voir aussi David Banon, « La lecture juive - une approche patiente », Pardès 32-33 (2002/1), p. 45-53 (46), <cairn.info > : « C'est [...] à travers l'écrit que le lecteur peut espérer - à force de labeur, au prix de discipline, de méthode, de grammaire, mais aussi avec l'intrépidité de l'imagination, bref à force de patience - dégager une gerbe de sens concernant Dieu, le monde et l'homme, en lisant/(ré) écoutant la parole coulée de manière fiable dans les lettres carrées. » 
pratiques autour du signe lui appartiennent totalement, mais Celan la pousse un peu plus vers ces autres aspects du passé collectif, d'un imaginaire construit autour des peuples actuels ou archaïques et des mots qui les incarnent. En invoquant l'Orient comme en s'intéressant à la chair du signe, elle met en relief « la nudité de la "matière-émotion" du langage », révèle l'intériorité de celui-ci, intériorité donc collective, partagée. ${ }^{10}$

Par ailleurs, les résonances entre les livres de Tellermann - comme à l'intérieur de chacun d'entre eux - sont nombreuses. Au niveau étymologique, le mot énigme se réfère à un parler allusif et au mot grec ainos, fable ou légende. Rares sont les poètes qui parviennent à poursuivre élégamment de recueil en recueil un seul et même récit, en l'occurrence une narration liée à la fable d'un devenir collectif qui comprend l'Antiquité. Le lecteur des poèmes de Tellermann participe au développement du récit en en déchiffrant l'énigme. Le moiré énonciatifl1 nous éclaire : la superposition de poétiques, le miroitement des voix derrière la grille de parole, l'invocation de distances paradoxalement proches, l'énigme dont on devine les lignes de force. On apprécie la variété de tons et s'habitue au registre soutenu, annonciateur. Les vers continuellement nuancés et la musique des reprises apportent de la joie, à la manière des textes anciens comme ceux des troubadours, où saisir les divers sens et les allusions retisser les messages communiqués - fait partie de l'écoute d'un texte gardant un rapport à l'oralité. On reconnaît un travail d'« orfèvre $»^{12}$ qui rend chatoyants

10 Mathieu Dubois, « Imaginaires extrêmes-orientaux dans la poésie contemporaine : enjeux d'une intériorité à retrouver », Esthétique et spiritualité II. Circulation des modèles en Europe, Baudouin Decharneux, Catherine Maignant et Myriam Watthee-Delmotte (sld), Fernelmont : EME, 2012, p. 245-6o (253). « Convoquer l'Orient signifie, pour les poètes, la voie d'un se dire soi, dans la nudité de la "matière-émotion" du langage ; c'est-à-dire révéler l'intériorité du sujet lyrique, sa "chair", en laquelle la Vie s'auto-révèle. À ce titre, la poésie moderne tient sa spécificité d'inventer, de manière toujours nouvelle et inépuisable, un langage de la "chair". Elle est tentative d'instituer [...] la parole d'un vivant, qui soit un appel à rompre les formes préfigurées du discours où la subjectivité se retire et devient illisible » (l'auteur souligne). Nous adaptons une expression de Patrick Née, qui parle de l'invocation dans des textes très courts de divers étants et actions et, en particulier, d'une mise en avant des verbes et de pronoms sujets ou bien absents, ou bien changeants et inattendus, alternant par exemple entre un Tu et un Vous. Ce mélange d'ambiguïté et d'immédiateté crée une «troublante polyphonie tissant une sorte de moire énonciative (l'auteur souligne). Il en résulte une étoffe où le grain du tissu est sans cesse dévié pour le rendre chatoyant, lumineux. Voir Patrick Née, «Une poétique de la voix », «Dossier Esther Tellermann » (éd. Jean-Baptiste Para), Éric Chevillard.Jean-Louis Giovannoni. Esther Tellermann, op. cit., p. 140-45 (143). Nous employons une métaphore voisine, le moiré, afin d'évoquer l'aspect ondoyant et lumineux de l'énigme : les effets de contraste lorsque deux grilles de parole sont superposées, obligeant le lecteur à rester attentif et à vouloir déchiffrer l'énigme dont les lignes de force lui apparaissent comme un méridien imaginaire. 
l'inachevé et le discontinu du discours. Le flux et le reflux de linconscient devient un plaisir, la fable ou la légende une fenêtre sur soi et les possibles du langage. Lorsqu'on lit tous les recueils de Guerre extrême à Un versant l'autre et considère leur enchaînement, on se sent pris tant dans le passage du temps que dans l'infini du désir. Pour Tellermann, «le multiple » que fait ainsi résonner l'écrivain se substitue à toute notion d'identité stable, lui permettant d'aller plus loin que le quotidien, d' « enquête $[\mathrm{r}]$ sur l'énigme du vivre et du mourir ».13 Que le poème de Celan nous mette "en présence d'une béance, d'un trou autour de quoi il tisse $\gg^{14}$ met particulièrement en évidence cet aspect ouvert de l'identité - peut-être celle de «l'âme ». ${ }^{15}$ Lire l'un après l'autre les livres de Tellermann en regard de ceux de Celan nous rappelle que cette ouverture identitaire se poursuit au niveau du souffle, du vivre et du mourir comme processus auquel rester attentif par le biais de mots qui veulent « luire ». ${ }^{16}$

Nous avons commencé cette étude en parlant d'un Je lyrique décentré, qui peut s'absenter du texte pour laisser la place aux mots et à l'Autre. Or, même si le sujet lyrique peut avoir chez Tellermann une présence discontinue, flottante, hésitante, brisée, celle qui parle privilégie des actes de parole intersubjectifs. Pour conclure, il convient de souligner cette constante de la trajectoire de l'auteur. Des trous dans le discours ne signalent pas seulement une absence, un redoublement ou un déplacement du sens, mais un vide à méditer à plusieurs, dans la fraîcheur de rites recommencés. Comme le dirait Tellermann, «Que les mots atteignent l'Autre est ce qui fait écriture ». ${ }^{17} \mathrm{Il}$ importe d'accéder au songe, à l'intersubjectif, au « Troisième » qui incarne des liens sacrés. Si l'écrivain «murmure une prière », elle y enserre «notre désarroi d'exister, peut-être $»^{18}$ : pour s'éveiller, naître, rester à l'écoute de la rumeur du monde et des voix qui nous tissent, accepter l'or et la boue. S'il est vrai que «l'accomplissement du deuil $»^{19}$ s'inscrit dans le temps, la beauté des textes tellermanniens tient à la fois des techniques employées qui en font des chants et des

13 Esther Tellermann dans Patrick Née, « Poème et identité : entretien avec Esther Tellermann », op. cit., p. 147 et p. 155 .

14 Ibid., p. 155 .

15 Ibid., p. 155 .

16 Paul Celan, «Strette», [Grille de parole], in John E. Jackson, Paul Celan: contre-parole et absolu poétique, op. cit., p. 92.

17 Correspondance avec l'auteur, 12 mai 2019, citée avec son aimable autorisation.

18 Ibid.

19 Myriam Watthee-Delmotte, Dépasser la mort: l'agir de la littérature, Paris : Actes Sud, 2019, p. 249-5o. Watthee-Delmotte mentionne «les trois séquences du deuil : commémorer le défunt, rejouer la mort et séparer le règne des vivants et des morts afin de relancer les endeuillés dans la vie » (19). Une spécificité chez Tellermann et Celan, c'est le fait de relier ces règnes, de parler en termes de suturer, ourler ou recoudre, afin que les morts soient eux aussi relancés dans la vie. 
offrandes sans cesse adressées aux morts comme aux vivants. C'est comme si l'habiter poétique maintenait un désaccord dans le temps et dans l'espace permettant aux morts de rester parmi nous. On ne s'attend pas à ce qu'il y ait un « retour à l'ordre ». ${ }^{20}$ À travers Tellermann, nous nous initions à un autre ordre du deuil, un ordre mythique : ce que Celan appelle « la prière / contre la prière $»,{ }^{21}$ contre l'oubli et dans l'espace vital des questions irrésolues, afin de ne pas laisser entièrement « cicatriser $»^{22}$ les blessures.

20 Denis Jeffrey, Jouissance du sacré: religion et postmodernité, Paris : Armand Colin, 1998, p. 125 .

21 Paul Celan, « Or », [Enclos du temps], Strette \& autres poèmes, op. cit., p. 127.

22 Paul Celan, « Voix », [Grille de parole], Choix de poèmes, op. cit., p. 129. 